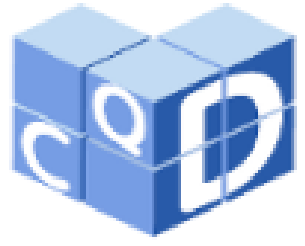

Revista Eletrônica

Paulista de Matemática

ISSN 2316-9664

Volume 21, dez. 2021

Jorge Corrêa de Araújo

Faculdade de Formação de Professores

Universidade do Estado do Rio de Janeiro

jcaraujo_55@yahoo.com.br

Rosa García Márquez

Faculdade de Formação de Professores

Universidade do Estado do Rio de Janeiro

rosagmarquez@yahoo.com.br

\section{Distribuição de temperaturas em cilindros circula- res infinitos: alguns casos de importância prática}

Temperature distribution in infinite circular cylinders: some cases of practical importance

\begin{abstract}
Resumo
Neste trabalho três casos de importância prática de distribuição de temperaturas em cilindros sólidos homogêneos ou ocos, finos e longos na geometria 1-D com respeito a variável radial são analisados. Os dois primeiros casos envolvem cilindros sólidos infinitos de aço-carbono. No primeiro cilindro é imposta uma condição de Newmann, enquanto no segundo cilindro, uma condição de Dirichlet do tipo parabólica é adotada na superfície. No terceiro caso é analisada a distribuição de temperatura em um cilindro oco formado por uma fina camada de alumínio com isolamento térmico na superfície externa. As soluções analíticas obtidas através de separação de variáveis e a transformada de Laplace mostram perfis de temperaturas consistentes com as condições propostas, e, portanto, adequadas a aplicações em engenharia.
\end{abstract}

Palavras-chave: Funções de Bessel. Funções de Bessel modificadas. Transformada de Laplace. Cilindro infinito.

\begin{abstract}
In this work, three cases of practical importance for temperature distribution in homogeneous or hollow, thin and long solid cylinders in 1-D geometry with respect to the radial variable are analyzed. The first two cases involve infinite solid carbon steel cylinders. In the first cylinder, a Newmann condition is imposed, while in the second cylinder, a parabolic Dirichlet condition is adopted on the surface. In the third case, the distribution of temperature in a hollow cylinder formed by a thin layer of aluminum with thermal insulation on the external surface is analyzed. The analytical solutions obtained through the separation of variables and the Laplace transform show temperature profiles consistent with the proposed conditions, and therefore suitable for engineering applications.
\end{abstract}

Keywords: Bessel functions. Modified Bessel functions. Laplace transform. Infinite circular cylinder. 


\section{Introdução}

Membranas circulares ou filmes finos têm muitas aplicações de interesse científico, como por exemplo, no experimento conhecido como "tubo de choque" em dinâmica dos gases (BIEZUNER, p. 76, 2010). De forma resumida, esse experimento consiste em um tubo cilíndrico longo e fino, separado por uma membrana delgada posicionada em uma seção transversal de interesse. Um gás é então colocado em cada lado com diferentes densidades, geralmente em repouso. Quando a membrana é subitamente retirada, observa-se o comportamento dos gases. Recentemente, um modelo simplificado do problema matemático associado a esse fenômeno foi analisado por Araújo e Márquez (2019, p. 163-180). Também em reatores nucleares com barras cilíndricas de elemento combustível como o Tório, onde ocorre a geração de calor, a temperatura em uma seção transversal do tubo envolta por um fino revestimento pode ser objeto de interesse de controle (INCROPERA; DEWITT; BERGMAN; LAVINE, 2008). Na biologia, a influência térmica na dinâmica de membranas altera segundo Ribeiro $(2012$, p. 3) a estrutura das membranas celulares podendo comprometer as atividades enzimáticas associadas aos processos de transporte. Embora uma análise 1-D não seja realista para o problema 3-D analisado, pois ela pode representar apenas uma possibilidade de retratar o fenômeno de interesse, Fox, Pritchard e McDonald (2015, p. 25) afirmam que muitos problemas na engenharia, mesmo uma análise 1-D é adequada para fornecer soluções aproximadas dentro da precisão requerida.

No entanto, a análise 1-D em um domínio finito, por exemplo, envolvendo a variável radial $r$ em coordenadas cilíndricas, duas superfícies devem ter condições de fronteira especificadas, e no caso em que há simetria, essas condições devem ser as mesmas. Em particular, os cilindros circulares infinitos, isto é, longos, $\frac{L}{r_{0}} \geq 10$, e finos, ou seja, $r_{0}$ pequeno, são adequados a uma análise unidimensional por atenderem ao requisito da simetria radial, $\partial_{r} T(r=0)=0$. Desse modo, tais problemas independem das variáveis angular $\theta$ e $z$ (ZILL; WRIGHT; CULLEN, 2011). Essas imposições de fronteira envolvem a condição de Dirichlet, $T_{S}=f(r, t)$, ou seja, a temperatura é prescrita na superfície $S$, Newmann, $\partial_{S} T=f(r, t)$, que é o fluxo de calor em $W / m^{2}$ que atravessa a superfície $S$ por unidade de área, e Cauchy ou convecção, $\partial_{S} T=h\left[T_{S}-T_{\infty}\right]$ que trata do deslocamento de calor de um meio para outro. Em geral, todas essas condições são lineares, podendo ser homogêneas ou não (HAHN; ÖZISIK, 2012, p. 1920).

Essas imposições estão presentes em diversos problemas físicos, como por exemplo, a temperatura prescrita na fronteira (Dirichlet), pode representar uma mudança de fase de uma substância que ocorre na superfície (evaporação/cozimento), ou como um fluxo de calor prescrito e controlado que segundo Hahn e Özisik (2012, p. 21) pode acontecer em aquecedores finos de fita elétrica fixos na superfície, que devido a sua baixa resistência de contato e submetido a um controle adequado, podem fornecer um fluxo de calor constante (Newmann). Já as condições de Cauchy são mais fáceis de realizar do ponto de vista físico, como por exemplo, um sistema de ventilação que desloca um fluido, como o ar mais frio de um ponto para outro. Embora naturais do ponto de vista físico, os problemas envolvendo fronteiras convectivas são

ARAÚJO, J. C. de.; MÁRQUEZ, R. G. Distribuição de temperaturas em cilindros circulares infinitos: alguns casos de importância prática. C.Q.D.- Revista Eletrônica Paulista de Matemática, Bauru, v. 21, p. 52-68, dez. 2021.

DOI: $10.21167 /$ cqdvol21202123169664jcargm5268 Disponível em: http://www.fc.unesp.br/departamentos/matematica/revista-cqd/ 
de grande complexidade (HAHN; ÖZISIK, 2012, p. 21). Apesar da importância do tema, são poucos os problemas de calor nessa geometria descritos com detalhes por diversos autores como, por exemplo, Carslaw e Jaeger (2011, p. 188-213), Churchill (1972, p. 244), Debnath e Bhatta (2015, p. 234), Trim (2013, p. 425) e Zill, Wright e Cullen (2011, p. 234) entre outros. Apenas Hahn e Özisik (2012, p. 156-157) trazem vários exemplos transientes na geometria 1D com diferentes condições de fronteira, mas que são discutidos eminentemente a luz do método de separação de variáveis.

Nosso objetivo é apresentar com detalhes três casos de interesse prático de distribuição de temperaturas em cilindros sólidos homogêneos ou ocos, finos e longos. O primeiro caso trata de uma distribuição de temperaturas em um cilindro sólido infinito de aço-carbono com condição de Newmann na superfície. Na verdade, esse problema é devido a Carslaw e Jaeger (2011, p. 328-329), cuja solução foi divulgada pelos autores de forma concisa. Esse problema é importante, pois aparece também em aplicações envolvendo difusão de substâncias como reportado por Crank (2011, p. 81). O segundo problema, trata de especificar sobre a superfície cilíndrica de aço-carbono uma temperatura parabólica, de modo a estudar o comportamento da distribuição de calor sobre a mesma, enquanto o terceiro caso analisa a distribuição de calor em um cilindro oco formado por uma fina camada de alumínio com isolamento térmico em sua superfície externa, e temperatura inicial variável com a posição do raio. Em todos os casos as condições de fronteira foram estendidas para situações mais gerais que as de temperaturas prescritas constantes na superfície, o que nesse sentido, impede que métodos, como o da transformada de Hankel finita (CINELLI, 1965, p. 539, 559) possa ser aplicado. Os resultados mostram que as soluções analíticas obtidas através de séries exponenciais no tempo, exigem poucos termos para a obtenção de perfis de temperaturas nos cilindros analisados, e desse modo, um melhor controle desses parâmetros com aplicações na engenharia pode ser proposto. Em especial na terceira aplicação, os perfis de temperaturas nessas posições foram obtidos com uma pequena perturbação no instante $t=0$. Os casos analisados nesse artigo podem ser estendidos no estudo de difusão de substâncias, pois segundo Crank (2011, p. 8) há um consenso entre a maioria dos cientistas e engenheiros que o fenômeno de fluxo de calor e difusão são basicamente os mesmos.

\section{Matemática e métodos}

A equação de condução de calor homogênea em regime transiente, e em um sistema 1D em coordenadas cilíndricas é dado pela equação (CARSLAW; JAEGER, 2011, p. 188)

$$
\frac{\partial^{2} T}{\partial r^{2}}+\frac{1}{r} \frac{\partial T}{\partial r}=\frac{1}{\kappa} \frac{\partial T}{\partial t}
$$

onde $\kappa$ é a difusividade térmica do material onde se dá o aquecimento.

\subsection{O problema de Sturm-Liouville em coordenadas cilíndricas}

Uma equação da forma

$$
\frac{d}{d x}\left[p(x) \frac{d y}{d x}\right]+[q(x)+\lambda w(x)] y=0,
$$


que satisfaz certas condições de fronteira em um intervalo $a \leq x \leq b$, sendo $p(x), q(x)$ e $r(x)$ funções contínuas nesse intervalo, com $p(x)$ diferençável, é chamada equação de Sturm Liouville. A busca de soluções não triviais para essa equação com as condições de fronteira nos extremos do intervalo é chamado problema de Sturm-Liouville (DAVIS, 1963, p. 64; SOTOMAYOR, 1979, p. 103). Tais soluções são denominadas autofunções, enquanto os $\lambda$ 's correspondentes são denominados autovalores. Esse problema em coordenadas cilíndricas é dado como na eq. (1) substituindo a variável $x$ por $r$, isto é,

$$
\frac{d}{d r}\left[p(r) \frac{d y}{d r}\right]+[q(r)+\lambda w(r)] y=0 .
$$

Em particular, se $p(r)=r, q(r)=-\frac{v}{r} \quad$ e $w(r)=r$, tem-se fazendo $y=R$, a equação diferencial ordinária na variável $r$ dada por

$$
\frac{d^{2} R}{d r^{2}}+\frac{1}{r} \frac{d R}{d r}+\left(\lambda^{2}-\frac{v^{2}}{r^{2}}\right) R=0
$$

onde geralmente $v=0,1,2 \ldots$

Essa equação sujeita as condições de fronteira homogêneas dadas por

$$
\begin{aligned}
& A_{1} \frac{d R}{d r}+A_{2} R=0, \quad r=a, \\
& B_{1} \frac{d R}{d r}+B_{2} R=0, \quad r=b
\end{aligned}
$$

é um problema de Sturm-Liouville em coordenadas cilíndricas (ou esféricas). As autofunções $R_{v}\left(r, \lambda_{n}\right)$, sob a condição de $\lambda>0$ ( HAHN; ÖZISIK, 2012, p. 43) que satisfazem a eq (3) e as condições dadas pela eq. (4) constituem um conjunto ortogonal de funções no espaço $C^{2}([a, b])$ com respeito ao produto interno dado por

$$
\langle f, g\rangle=\int_{a}^{b} r f(r) g(r) d r,
$$

sendo a norma quadrática de cada autofunção dada por Hahn e Özisik (2012, p. 51)

$$
N\left(\lambda_{n}\right)=N\left[R_{v}\left(r, \lambda_{n}\right)\right]=\int_{a}^{b} r R_{v}^{2}\left(r, \lambda_{n}\right) d r .
$$

Segundo Davis (1963, p. 67) toda função $R$ limitada e integrável no espaço $C^{2}([a, b])$ que satisfaz as condições de fronteiras dadas pela eq (5) pode ser expandida em uma série que converge ponto a ponto nesse intervalo, isto é,

$$
R(r)=\sum_{n=1}^{\infty} C_{n} R_{v}\left(r, \lambda_{n}\right)
$$

onde $C_{n}=\frac{1}{N\left(\lambda_{n}\right)} \int_{a}^{b} r R(r) R_{v}\left(r, \lambda_{n}\right) d r ; \quad n=1, \ldots$.

A eq. (4) é chamada de equação de Bessel e tem como solução mais geral a equação dada por (HAHN; ÖZISIK, 2012, p. 48)

$$
R(r)=c_{1} J_{v}(\lambda r)+c_{2} Y_{v}(\lambda r)
$$


onde $J_{v}(\lambda r)$ e $Y_{v}(\lambda r)$ são respectivamente as funções de Bessel de primeira e segunda espécie de ordem $v$. Os autovalores $\lambda$ são obtidos por meio de equações transcendentais oriundas das condições de fronteira especificadas. A equação de Bessel modificada surge da eq (3) na forma (HAHN; ÖZISIK, 2012, p. 55)

$$
\frac{d^{2} R}{d r^{2}}+\frac{1}{r} \frac{d R}{d r}-\left(\eta^{2}+\frac{v^{2}}{r^{2}}\right) R=0
$$

cuja solução geral é dada por

$$
R(r)=c_{1} I_{v}(\eta r)+c_{2} K_{v}(\eta r),
$$

onde $I_{v}(\eta r)$ e $K_{v}(\eta r)$ são respectivamente as funções de Bessel modificadas de primeira e segunda espécie de ordem $v$. Tais funções não satisfazem ao critério de Sturm-Liouville, pois, $w(r)=-r$. Por isso não são ortogonais sob o produto interno anteriormente definido e desse modo o método de separação de variáveis não pode ser aplicado.

\subsection{Método de Separação de Variáveis}

Um método padrão para obter uma solução para a eq. (1) é assumir que as variáveis são separáveis, isto é,

$$
T(r, t)=G(r) H(t),
$$

onde $G$ e $H$ são só funções de $r$ e $t$ respectivamente. A substituição da eq. (12) na eq. (1) resulta em duas equações diferenciais ordinárias independentes na forma HAHN; OZISIK, 2012, p. 156)

$$
\frac{d^{2} G}{d r^{2}}+\frac{1}{r} \frac{d G}{d r}=\frac{1}{\kappa H} \frac{d H}{d t}=-\lambda^{2}
$$

ou, seja

$$
\begin{gathered}
\frac{1}{\kappa H} \frac{d H}{d t}=-\lambda^{2}, \\
\frac{d^{2} G}{d r^{2}}+\frac{1}{r} \frac{d^{2} G}{d r^{2}}+\lambda^{2} G=0 .
\end{gathered}
$$

A eq (14) pode ser resolvida diretamente por separação de variáveis para obter,

$$
H(t)=c_{1} e^{-\kappa \lambda^{2} t},
$$

enquanto a EDO na variável $r$ é uma equação de Bessel de ordem $v=0$, cuja solução geral é dada pela eq (9) na forma

$$
G(r)=c_{2} J_{0}(\lambda r)+c_{3} Y_{0}(\lambda r)
$$

onde as constantes poderão ser eliminadas por meio de condições iniciais adequadas. Portanto, uma solução geral da eq (1) é dada pelo produto das soluções individuais dadas pelas eqs. (16) e (17), isto é,

$$
T(r, t)=c_{1} e^{-\kappa \lambda^{2} t}\left[c_{2} J_{0}(\lambda r)+c_{3} Y_{0}(\lambda r)\right]
$$


ou omitindo as constantes,

$$
T(r, t)=e^{-\kappa \lambda^{2} t} G_{0}(r, \lambda) .
$$

Como para cada $\lambda_{n}>0$, podemos associar uma autofunção $G_{v}\left(r, \lambda_{n}\right)$ e desde que a eq. (1) é linear, a solução mais geral da eq. (1) será obtida pela soma de todos os produtos das eqs. (16) e (17)

$$
T(r, t)=\sum_{n=1}^{\infty} c_{n} e^{-\kappa \lambda^{2} t} G_{0}\left(r, \lambda_{n}\right)
$$

\subsection{Método da Transformada de Laplace}

Se a função $F(t)$ é definida para todos os valores positivos na variável $t$, uma nova função $\bar{F}(p)$ é obtida no parâmetro $p$, isto é,

$$
L\{F(t)\}=\bar{F}(p)=\int_{0}^{\infty} e^{-p t} F(t) d t .
$$

Essa nova função é chamada de transformada de Laplace (CHURCHILL, 1972, p. 3) e pode ser aplicada na eq (1) para obter uma equação diferencial ordinária subsidiaria. De fato,

$$
L\left\{\frac{\partial^{2} T}{\partial r^{2}}+\frac{1}{r} \frac{\partial T}{\partial r}=\frac{1}{\kappa} \frac{\partial T}{\partial t}\right\} \Leftrightarrow L\left\{\frac{\partial^{2} T}{\partial r^{2}}\right\}+L\left\{\frac{1}{r} \frac{\partial T}{\partial r}\right\}-L\left\{\frac{1}{\kappa} \frac{\partial T}{\partial t}\right\}=0,
$$

ou

$$
\frac{d^{2} \bar{T}}{\partial r^{2}}+\frac{1}{r} \frac{d \bar{T}}{d r}-q^{2} \bar{T}=0
$$

onde $q=\sqrt{\frac{p}{\kappa}}$ e se a condição inicial é $T(r, 0)=0$ tem-se $L\left\{\frac{1}{\kappa} \frac{\partial T}{\partial t}\right\}=\frac{p}{\kappa} \bar{T}$. Uma vez obtida à solução $\bar{T}=\bar{T}(r, p)$ na variável $r$ com o parâmetro $p$, pode ser aplicado à transformada inversa de Laplace (CHURCHILL, 1972, p. 14) para obter a solução do problema original, isto é,

$$
L^{-1}\{\bar{T}(r, p)\}=T(r, p)
$$

A transformada inversa de Laplace é definida pela integral complexa segundo Debnath e Bhatta (2015, p. 173) por

$$
L^{-1}\{\bar{f}(p)\}=f(t)=\frac{1}{2 \pi i} \int_{c-i \infty}^{c+i \infty} e^{p t} \bar{f}(p) d p
$$

O caminho de integração é uma linha reta $L$ no plano complexo $p$ com equação $p=c+i R,-\infty<R<\infty, \operatorname{Re}(p)=c$, onde $c$ é escolhido de modo que todas as singularidades do integrando da eq (25) estejam à esquerda da reta $L$. Esse caminho de integração é chamado caminho de Bromwich (DEBNATH; BHATTA, 2015, p. 173) e será aqui assumido nesse artigo. A eq (25) pode então ser calculada por meio da eq. (3.7.9) proposta por Debnath e Bhatta (2015, p. 173) na forma

$$
L^{-1}\{\bar{f}(p)\}=f(t)=\frac{1}{2 \pi i} \int_{c-i \infty}^{c+i \infty} e^{p t} \bar{f}(p) d p=\sum_{n=1}^{\infty} \rho_{n}(t),
$$


onde $\rho_{n}(t)$ são os resíduos nos polos de $\bar{f}(p)$. Se $\bar{g}(p)=e^{p t} \bar{f}(p)$ tem um polo de ordem $n$ em $p=z$, o resíduo é dado pela eq. (3.7.10) reportada por Debnath e Bhatta (2015, p. 173).

$$
\rho_{n}(t)=\lim _{p \rightarrow z} \frac{1}{(n-1) !} \frac{d^{n-1}}{d p^{n-1}}(p-z) \bar{g}(p) .
$$

Outro resultado importante (CHURCHILL 1972, p. 209) diz que em particular, se $f(z)=\frac{g(z)}{q(z)}$, onde $g$ e $q$ são analíticas em $p_{n}$ e $q\left(p_{n}\right)=0$, enquanto $g\left(p_{n}\right) \neq 0$ e $\frac{d q}{d z}\left(p_{n}\right) \neq 0$, então

$$
\rho_{n}(t)=\frac{g\left(p_{n}\right)}{\frac{d q}{d z}\left(p_{n}\right)} e^{p_{n} t}
$$

\section{Resultados e discussões}

Nessa seção são apresentados três casos de problemas térmicos com aplicações em engenharia, envolvendo a distribuição de temperaturas em cilindros unidimensionais na variável radial, longos e finos com não homogeneidades presentes nas condições de contorno. Portanto, as soluções analíticas aqui apresentadas foram detalhadas de forma didática, o que facilita o seu entendimento por um leitor interessado.

\subsection{Caso I}

A Fig. 1 mostra um longo cilindro circular de aço-carbono, $0 \leq r<a$, com temperatura inicial nula, submetido a um fluxo de calor constante $F_{0}$ na superfície.

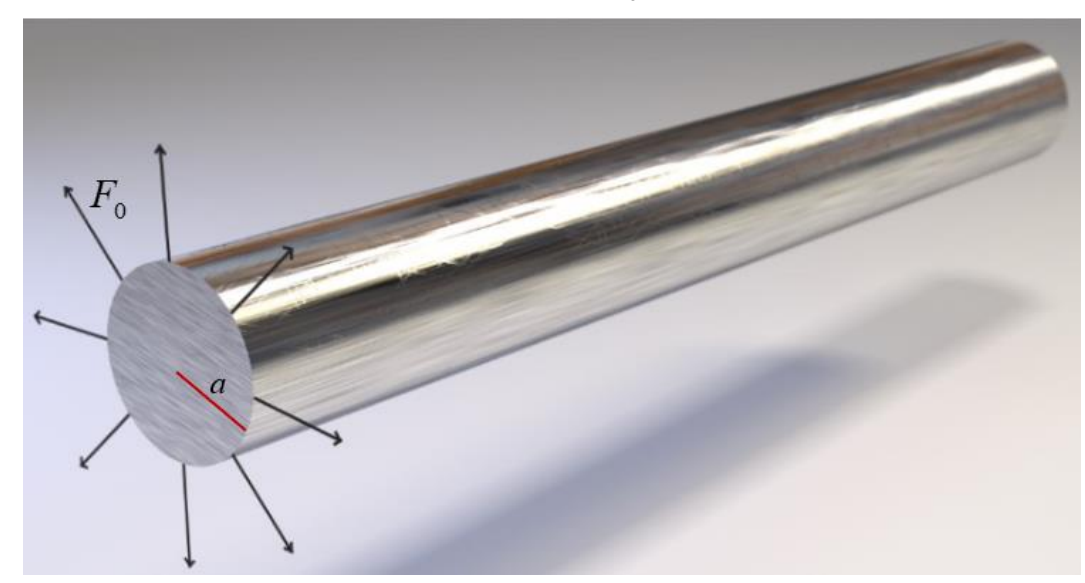

Figura 1. Cilindro longo e fino com fluxo de calor constante na superfície $r=a$.

A formulação matemática desse problema é dada por

$$
\frac{\partial^{2} T}{\partial r^{2}}+\frac{1}{r} \frac{\partial T}{\partial r}=\frac{1}{\kappa} \frac{\partial T}{\partial t}, \quad 0 \leq r<a \quad t>0
$$




$$
\begin{aligned}
& C F 1:-K \frac{\partial T}{\partial r}(a, t)=F_{0}, \\
& C F 2: \lim _{r \rightarrow 0} T(r, t)<\infty, \\
& \text { C.I: } T(r, 0)=0, \quad 0 \leq r \leq a .
\end{aligned}
$$

Aqui CF indica a condição de fronteira, enquanto CI a condição inicial. Essa notação será usando ao longo do texto.

Aplicando a Transformada de Laplace nas eqs (29) e (30) tem-se uma EDO na variável $r$ sujeita as condições dadas por

$$
\begin{aligned}
& \frac{d^{2} \bar{T}}{d r^{2}}+\frac{1}{r} \frac{d \bar{T}}{d r}-q^{2} \bar{T}=0, \quad 0 \leq r<a . \\
& C F 1:-K \frac{d \bar{T}}{d r}(a, p)=\frac{F_{0}}{p}, \\
& C F 2: \lim _{r \rightarrow 0} \bar{T}(r, p)<\infty,
\end{aligned}
$$

onde $q^{2}=\frac{p}{\kappa}$. A EDO está na forma da eq (10) com $v=0$, portanto é uma equação de Bessel modificada de ordem zero, e como $K_{0}(q r \rightarrow 0) \rightarrow \infty$, essa função devido a condição CF2 tem que ser excluída da solução geral. Desse modo, da eq (11) e CF1 e usando o fato que $\frac{d}{d r} I_{0}(q r)=q I_{1}(q r)$ tem-se

$$
c_{1}=-\frac{F_{0}}{K} \sqrt{\frac{\kappa}{p}} \frac{1}{I_{1}(q a)} .
$$

Logo,

$$
\bar{T}=-\frac{F_{0}}{K} \frac{\kappa^{\frac{1}{2}}}{p^{\frac{3}{2}}} \frac{I_{0}(q r)}{I_{1}(q a)} .
$$

Da eq (33) e do teorema da inversão dado pela eq (26) resulta

$$
T=\frac{1}{2 \pi i} \int_{c-i \infty}^{c+i \infty} e^{\lambda t} \bar{T}(\lambda) d \lambda,
$$

onde

$$
\bar{T}=-\frac{F_{0}}{K} \frac{\kappa^{\frac{1}{2}}}{\lambda^{\frac{3}{2}}} \frac{I_{0}(\mu r)}{I_{1}(\mu a)},
$$

sendo $\mu=\sqrt{\frac{\lambda}{\kappa}}$, notação sugerida por Carslaw e Jaeger (2011, p. 328). Estamos admitindo que a integração seja sobre um caminho de Bromwich. As singularidades de $\bar{T}$ são os zeros de $I_{1}(\mu a)$, onde em particular, $\lambda_{0}=0$ não anula $I_{1}(\mu a)$. Fazendo $\mu=i \alpha_{s}$, tem-se $\lambda=-\kappa \alpha_{s}^{2}$. Daí resulta, $I_{1}(\mu a)=I_{1}\left(i \alpha_{s} a\right)=i J_{1}\left(\beta_{s}\right)$, onde $\beta_{s}=a \alpha_{s}$. Desse modo, os zeros de $I_{1}(\mu a)$, são os 
$\lambda_{s}=-\kappa \alpha_{s}^{2}$ onde $\pm \alpha_{s}$, sendo $s=1,2, \ldots$ as raízes positivas da equação $J_{1}(\alpha)=0$. Para obter os resíduos $\rho_{s}(t)$ nesses polos devemos calcular $\left.\frac{\kappa^{\frac{1}{2}}}{p^{\frac{3}{2}}} \frac{d I_{1}}{d \lambda}(\mu a)\right|_{\lambda=-\kappa \alpha_{s}^{2}} ^{\mu=i \alpha_{s}}$.

$$
\left.\frac{\kappa^{\frac{1}{2}}}{p^{\frac{3}{2}}} \frac{d I_{1}}{d \lambda}(\mu a)\right|_{\lambda=-\kappa \alpha_{s}^{2}} ^{\mu=i \alpha_{s}}=-\kappa \alpha_{s}^{2} i \alpha_{s}\left[-\frac{a}{2 \kappa \alpha_{s}} i J_{0}\left(\beta_{s}\right)\right]=-\frac{1}{2 a} \beta_{s}^{2} J_{0}\left(\beta_{s}\right)(\neq 0)
$$

A eq (35) pode ser posta na forma $\bar{T}=\frac{g(\lambda)}{q(\lambda)}$, onde $g(\lambda)=-\frac{F_{0}}{K} \frac{\kappa^{\frac{1}{2}}}{\lambda^{\frac{3}{2}}} I_{0}(\mu r)$ e $q(\lambda)=I_{1}(\mu a)$ de modo a usarmos as eqs (28) e (36) para obter

$$
\rho_{s}(t)=\operatorname{Res}\left[e^{\lambda t} \bar{T}, \lambda_{s}\right]=\frac{g\left(\lambda_{s}\right)}{\frac{d q}{d \lambda}} e^{\lambda_{s} t}=\frac{F_{0}}{K} \frac{2 a}{\beta_{s}^{2} J_{0}\left(\beta_{s}\right)} I_{0}\left(i \alpha_{s} r\right) e^{-\kappa \alpha_{s}^{2} t}=2 a \frac{F_{0}}{K} \frac{J_{0}\left(\frac{\beta_{s} r}{a}\right)}{\beta_{s}^{2} J_{0}\left(\beta_{s}\right)} e^{-\kappa \alpha_{s}^{2} t},
$$

com $s=1,2, \ldots$

Para investigar o polo $\lambda_{s=0}=0$ que aparece na eq (35) vamos desenvolver a expansão de Laurent de $e^{\lambda t} \bar{T}$ em torno desse ponto.

$$
\frac{I_{0}(\mu r)}{I_{1}(\mu a)}=\frac{1+\left(\frac{1}{2} \mu r\right)^{2}+\frac{1}{4}\left(\frac{1}{2} \mu r\right)^{4}+\ldots}{\left(\frac{1}{2} \mu a\right)^{2}+\frac{1}{2}\left(\frac{1}{2} \mu r\right)^{3}+\ldots} \Rightarrow \frac{\kappa^{\frac{1}{2}}}{\lambda^{\frac{3}{2}}} \frac{I_{0}(\mu r)}{I_{1}(\mu a)}=\frac{2 \kappa}{a \lambda^{2}}+\frac{\lambda r^{2}}{2 a}-\frac{1}{4} \lambda a+\ldots
$$

Portanto, existe um polo duplo em $\lambda_{s=0}=0$. Da eq (27) obtemos

$$
\rho_{0}(t)=\lim _{\lambda \rightarrow 0}\left[\frac{d}{d \lambda} \lambda^{2} e^{\lambda t} \bar{T}\right]=-\frac{F_{0}}{K}\left[\frac{2 \kappa t}{a}+\frac{r^{2}}{2 a}-\frac{1}{4} a\right] .
$$

Das eqs (34), (37) e (39) resulta

$$
T(r, t)=\rho_{0}(t)+\sum_{s=1}^{\infty} \rho_{s}(t)=-\frac{F_{0} a}{K}\left\{\frac{2 \kappa t}{a^{2}}+\frac{r^{2}}{2 a^{2}}-\frac{1}{4}-2 \sum_{s=1}^{\infty} e^{-\kappa \beta_{s}^{2} t / a^{2}} \frac{J_{0}\left(\frac{\beta_{s} r}{a}\right)}{\beta_{s}^{2} J_{0}\left(\beta_{s}\right)}\right\} .
$$

A eq (40) coincide com a eq (11), reportada de modo conciso por Carslaw e Jaeger (2011, p. 329), isto é, a solução analítica desse problema de difusão de calor apresentada pelos referidos autores não deixa claro os procedimentos intermediários para a obtenção dessa solução.

Se a C.I dada pela eq (30) for $T(r, t=0)=V$, a solução para a distribuição de calor é obtida diretamente da eq (40) adicionando o termo constante $V$, mediante a translação $T-V$. 
A Fig. 2 mostra o perfil de temperaturas em diferentes instantes de tempo no cilindro de raio $a=0.1 \mathrm{~m}$ com temperatura inicial $V=10^{\circ} \mathrm{C}$ e utilizando os seguintes parâmetros, em particular $K e \kappa$ adequados ao aço-carbono. $K=63.4 \mathrm{~W} / \mathrm{m}^{\circ} \mathrm{C} ; \kappa=18.8 \times 10^{-6} \mathrm{~m}^{2} / \mathrm{s}$ e $F_{0}=20 \mathrm{~W} / \mathrm{m}^{2}$, onde o tempo foi obtido em minutos mediante a multiplicação do termo $1 / s$ por 60.

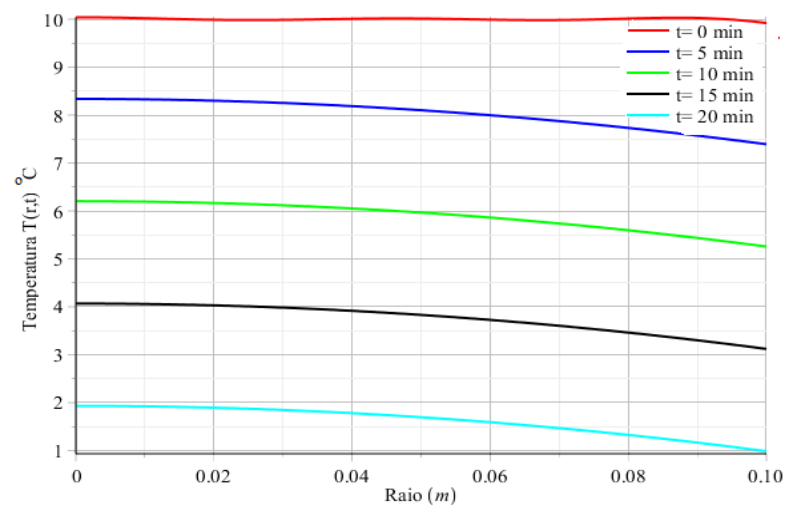

Figura 2. Perfil de temperaturas no cilindro circular infinito com $T(r, 0)=10^{\circ} \mathrm{C}$ e $-K \frac{\partial T}{\partial r}(a, t)=20 \mathrm{~W} / \mathrm{m}^{2}$.

Nesse problema foram utilizadas as quatro primeiras raízes positivas da equação $J_{1}(\alpha)=0$, a saber: $\alpha_{1}=3.831, \alpha_{2}=7.015, \alpha_{3}=10.173$ e $\alpha_{4}=13.323$.

\subsection{Caso II}

A Fig. 3 mostra um cilindro circular infinito, fino de aço-carbono, $0 \leq r<a=0.1 m$, com temperatura inicial nula, submetido a uma temperatura parabólica na superfície.

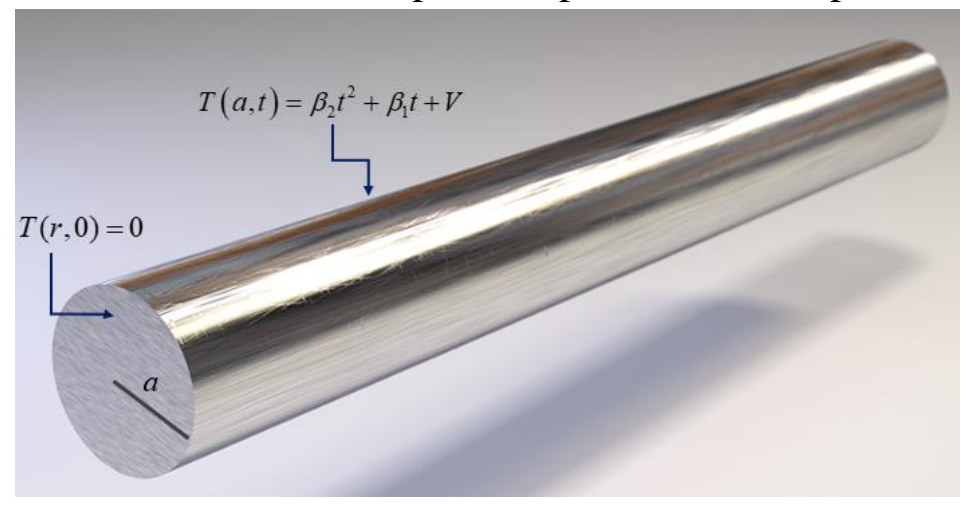

Figura 3. Cilindro circular infinito de aço-carbono

A formulação matemática desse problema é dada por

$$
\begin{aligned}
& \frac{\partial^{2} T}{\partial r^{2}}+\frac{1}{r} \frac{\partial T}{\partial r}=\frac{1}{\kappa} \frac{\partial T}{\partial t}, \quad 0 \leq r<a \quad t>0 . \\
& C F 1: T(a, t)=\beta_{2} t^{2}+\beta_{1} t+V, \\
& C F 2: \lim _{r \rightarrow 0} T(r, t)<\infty, \\
& \text { C.I: } T(r, 0)=0, \quad 0 \leq r \leq a .
\end{aligned}
$$


Aqui $\beta_{2}=\frac{\beta}{2}$ por razões de conveniência para a aplicação da transformada de Laplace. Aplicando a transforma de Laplace nas eqs (41) e (42) obtém-se uma EDO na variável $r$ com condições de fronteira dadas por

$$
\begin{aligned}
& \frac{d^{2} \bar{T}}{d r^{2}}+\frac{1}{r} \frac{d \bar{T}}{d r}-q^{2} \bar{T}=0, \quad 0 \leq r<a . \\
& C F 1: \lim _{r \rightarrow a} \bar{T}(r, p)=\frac{\beta}{p^{3}}+\frac{\beta_{1}}{p^{2}}+\frac{V}{p} \\
& C F 2: \lim _{r \rightarrow 0} \bar{T}(r, p)<\infty,
\end{aligned}
$$

De modo análogo ao caso I, essa EDO é uma equação de Bessel modificada de ordem zero, e como $K_{0}(q r \rightarrow 0) \rightarrow \infty$, essa função devido à condição CF2, tem que ser excluída da solução geral. Desse modo, da eq. (11) tem-se

$$
\bar{T}(r, p)=c_{1} I_{0}(q r) \text {. }
$$

Da eq (44) e de CF1 dada pela eq. (43) resulta

$$
\bar{T}(r, p)=\left(\frac{\beta}{p^{3}}+\frac{\beta_{1}}{p^{2}}+\frac{V}{p}\right) \frac{I_{0}(q r)}{I_{0}(q a)} .
$$

Da eq (45) e do teorema da inversão dado pela eq (26) resulta

$$
T=\frac{1}{2 \pi i} \int_{c-i \infty}^{c+i \infty} e^{\lambda t} \bar{T}(\lambda) d \lambda
$$

onde

$$
\bar{T}=\left(\frac{\beta}{\lambda^{3}}+\frac{\beta_{1}}{\lambda^{2}}+\frac{V}{\lambda}\right) \frac{I_{0}(\mu r)}{I_{0}(\mu a)}
$$

sendo $\mu=\sqrt{\frac{\lambda}{\kappa}}$. Estamos admitindo que a integração seja sobre um caminho de Bromwich. As singularidades de $\bar{T}$ são os zeros de $I_{0}(\mu a)$, e os polos em zero que aparecem no denominador de $\bar{T}$. A forma da eq (35) permite a seguinte decomposição linear, isto é,

$$
\bar{T}=\bar{T}_{1}+\bar{T}_{2}+\bar{T}_{3},
$$

onde

$$
\overline{T_{1}}=\frac{\beta}{\lambda^{3}} \frac{I_{0}(q r)}{I_{0}(q a)}, \quad \bar{T}_{2}=\frac{\beta_{1}}{\lambda^{2}} \frac{I_{0}(q r)}{I_{0}(q a)} \quad \text { e } \quad \bar{T}_{3}=\frac{V}{\lambda} \frac{I_{0}(q r)}{I_{0}(q a)} .
$$

As transformadas inversas de Laplace sobre $\bar{T}_{2} e \bar{T}_{3}$ podem ser obtidas diretamente das equações (7) e (8) reportadas por Carslaw e Jaeger (2011, p. 327-328) na forma

$$
T_{2}=\beta_{1}\left(t-\frac{a^{2}-r^{2}}{4 \kappa}\right)+\frac{2 \beta_{1}}{a \kappa} \sum_{n=1}^{\infty} e^{-\kappa \alpha_{n}^{2} t} \frac{J_{0}\left(\alpha_{n} r\right)}{\alpha_{n}^{3} J_{1}\left(a \alpha_{n}\right)} \text { e } T_{3}=V-\frac{2 V}{a} \sum_{n=1}^{\infty} e^{-\kappa \alpha_{n}^{2} t} \frac{J_{0}\left(\alpha_{n} r\right)}{\alpha_{n} J_{1}\left(a \alpha_{n}\right)},
$$

onde $\alpha_{n}$ são as raízes positivas da equação $J_{0}(a \alpha)=0$. Fazendo $\mu=i \alpha$, tem-se $\lambda=-\kappa \alpha^{2}$. Daí, e usando a eq (26) do Apêndice III de Carslaw e Jaeger (2011, p. 490) podemos escrever $I_{0}(\mu a)=I_{0}(i a \alpha)=J_{0}(a \alpha)$. 
Desse modo, os zeros de $I_{0}(\mu a)$ são os $\lambda=-\kappa \alpha_{n}^{2}$, onde $\pm \alpha_{n}$, com $n=1,2, .$. são as raízes da equação $J_{0}(a \alpha)=0$. Para obter os resíduos nesses polos devemos calcular $\left.\lambda^{3} \frac{d I_{0}}{d \lambda}(\mu a)\right|_{\lambda=-\kappa \alpha_{s}^{2}} ^{\mu=i \alpha_{s}}$ ou seja, usando a propriedade $I_{1}\left(i a \alpha_{n}\right)=J_{1}\left(a \alpha_{n}\right)$ tem-se

$$
\left.\lambda^{3} \frac{d I_{0}}{d \lambda}(\mu a)\right|_{\lambda=-\kappa \alpha_{n}^{2}} ^{\mu=i \alpha_{n}}=\frac{\lambda^{3} a I_{1}\left(i a \alpha_{n}\right) \lambda^{-\frac{1}{2}}}{2 \kappa^{\frac{1}{2}}}=-\frac{a}{2} \kappa^{2} \alpha_{n}^{-5} J_{1}\left(a \alpha_{n}\right),
$$

Os resíduos nesses polos podem ser calculados usando a eq (28) para obter

$$
\rho_{n}(t)=-\frac{2 \beta}{a \kappa^{2}} \frac{J_{0}\left(r \alpha_{n}\right)}{\alpha_{n}^{5} J_{1}\left(a \alpha_{n}\right)} e^{-\kappa \alpha_{n}^{2} t}, \quad n=1,2, \ldots
$$

Para analisar o resíduo no $\lambda_{0}=0$ de ordem três que aparece na expressão de $\bar{T}_{1}$ vamos expandir $\bar{T}_{1}$ em série de Laurent em torno desse polo.

$$
I_{0}(\mu r)=1+\frac{1}{2}(\mu r)^{2}+\frac{3}{64}(\mu r)^{4}+\ldots \quad \text { e } \quad \frac{1}{I_{0}(\mu a)}=1-\frac{1}{4}(\mu a)^{2}+\frac{3}{64}(\mu a)^{4}-\ldots
$$

Assim,

$$
\frac{I_{0}(\mu r)}{I_{0}(\mu a)}=\left[1+\frac{1}{2}(\mu r)^{2}+\frac{3}{64}(\mu r)^{4}+\ldots\right]\left[1-\frac{1}{4}(\mu a)^{2}+\frac{3}{64}(\mu a)^{4}-\ldots\right] .
$$

Das eqs (27), (49), (54) tem-se

$$
\rho_{0}(t)=\lim _{\lambda \rightarrow 0}\left[\frac{1}{2} \frac{d^{2}}{d \lambda^{2}} \lambda^{2} e^{\lambda t} \bar{T}\right]=\frac{\beta}{2}\left\{t^{2}-\left(\frac{a^{2}-r^{2}}{2 \kappa}\right) t-\frac{a^{2} r^{2}}{8 \kappa^{2}}+\frac{r^{4}}{32 \kappa^{2}}+\frac{3 a^{4}}{32 \kappa^{2}}\right\} .
$$

Das eqs (49), (52) e (55) tem-se

$$
T_{1}=\frac{\beta}{2}\left\{t^{2}-\left(\frac{a^{2}-r^{2}}{2 \kappa}\right) t-\frac{a^{2} r^{2}}{8 \kappa^{2}}+\frac{r^{4}}{32 \kappa^{2}}+\frac{3 a^{4}}{32 \kappa^{2}}\right\}-\frac{2 \beta}{a \kappa^{2}} \frac{J_{0}\left(r \alpha_{n}\right)}{\alpha_{n}^{5} J_{1}\left(a \alpha_{n}\right)} e^{-\kappa \alpha_{n}^{2} t} .
$$

Das eqs (48), (50) e (56) resulta a solução do problema de calor na forma

$$
\begin{aligned}
T= & V-\frac{2 V}{a} \sum_{n=1}^{\infty} e^{-\kappa \alpha_{n}^{2} t} \frac{J_{0}\left(\alpha_{n} r\right)}{\alpha_{n} J_{1}\left(a \alpha_{n}\right)}+\beta_{1}\left(t-\frac{a^{2}-r^{2}}{4 \kappa}\right)+\frac{2 \beta_{1}}{a \kappa} \sum_{n=1}^{\infty} e^{-\kappa \alpha_{n}^{2} t} \frac{J_{0}\left(\alpha_{n} r\right)}{\alpha_{n}^{3} J_{1}\left(a \alpha_{n}\right)}+ \\
& +\frac{\beta}{2}\left\{t^{2}+\left(\frac{-a^{2}+r^{2}}{2 \kappa}\right) t-\frac{a^{2} r^{2}}{8 \kappa^{2}}+\frac{r^{4}}{32 \kappa^{2}}+\frac{3 a^{4}}{32 \kappa^{2}}\right\}-\frac{2 \beta}{a \kappa^{2}} \frac{J_{0}\left(r \alpha_{n}\right)}{\alpha_{n}^{5} J_{1}\left(a \alpha_{n}\right)} e^{-\kappa \alpha_{n}^{2} t} .
\end{aligned}
$$

Se a C.I dada pela eq (42) é substituída por $T(r, 0)=T_{0}$ basta fazer a translação $T-T_{0}$ para obter

$$
\begin{aligned}
T= & V-T_{0}-\frac{2\left(V-T_{0}\right)}{a} \sum_{n=1}^{\infty} e^{-\kappa \alpha_{n}^{2} t} \frac{J_{0}\left(\alpha_{n} r\right)}{\alpha_{n} J_{1}\left(a \alpha_{n}\right)}+\beta_{1}\left(t-\frac{a^{2}-r^{2}}{4 \kappa}\right)+\frac{2 \beta_{1}}{a \kappa} \sum_{n=1}^{\infty} e^{-\kappa \alpha_{n}^{2} t} \frac{J_{0}\left(\alpha_{n} r\right)}{\alpha_{n}^{3} J_{1}\left(a \alpha_{n}\right)}+ \\
& \frac{\beta}{2}\left\{t^{2}+\left(\frac{-a^{2}+r^{2}}{2 \kappa}\right) t-\frac{a^{2} r^{2}}{8 \kappa^{2}}+\frac{r^{4}}{32 \kappa^{2}}+\frac{3 a^{4}}{32 \kappa^{2}}\right\}-\frac{2 \beta}{a \kappa^{2}} \sum_{n=1}^{\infty} e^{-\kappa \alpha_{n}^{2} t} \frac{J_{0}\left(r \alpha_{n}\right)}{\alpha_{n}^{5} J_{1}\left(a \alpha_{n}\right)} .
\end{aligned}
$$

Em particular, se $T_{0}=0, \mathrm{e}$ 


$$
\beta_{2}=\beta=0, \beta_{1}=0 \Rightarrow T=T_{3} \text { e } \beta_{2}=\beta=0, V=0 \Rightarrow T=T_{2},
$$

Ou seja, a eq (58) generaliza as condições propostas por Carslaw e Jaeger (2011, p. 327328) para temperatura prescrita na superfície do tipo linear. A Fig. 4 mostra o perfil parabólico de temperaturas em posições específicas no cilindro de raio $a=0.1$ (m) utilizando os seguintes parâmetros, em particular, $K e \kappa$ adequados ao aço-carbono, $K=63.4 \mathrm{~W} / \mathrm{m}^{\circ} \mathrm{C}$, $\kappa=18.8 \times 10^{-6} \mathrm{~m}^{2} / \mathrm{s}$. O tempo foi obtido em minutos mediante a multiplicação do termo $1 / \mathrm{s}$ por 60.
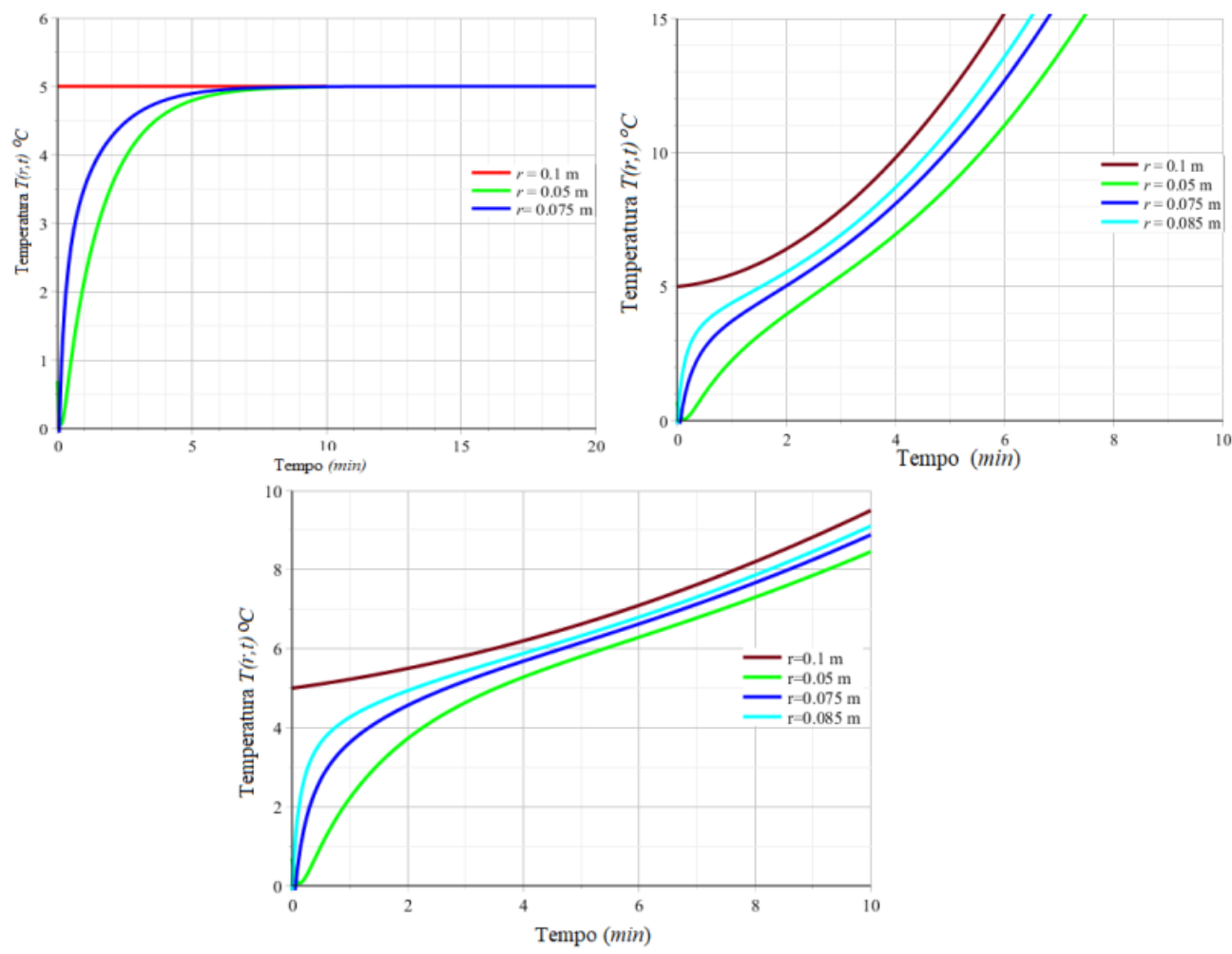

Figura 4. Perfil de temperaturas no cilindro sólido circular: (a) $T(r, 0)=T_{0}=5^{\circ} C, \beta=10^{-3} e \beta_{1}=0$, (b) $T(t, 0)=T_{0}=0^{\circ} C, T(a, t)=V=5^{\circ} C, \beta=0.5$ e $\beta_{1}=\gamma=0$ (c) $T(a, t)=V=10, T(r, 0)=T_{0}=5^{\circ} C$, $T(r, 0)=T_{0}=5^{\circ} C, \quad \beta=0.05$ e $\beta_{1}=0.025$.

Nesse problema foram utilizadas as quatro primeiras raízes positivas da equação $J_{0}(a \alpha)=0$, a saber: $\alpha_{1}=24.048, \alpha_{2}=55.200, \alpha_{3}=86.537$ e $\alpha_{4}=117.915$.

\subsection{Caso III}

A Fig. 5 mostra uma seção transversal de um cilindro oco de alumínio de fina espessura, com $a=0,02 \leq r<b=0,03(\mathrm{~m})$. Vamos admitir que a temperatura do alumínio seja constante em $r=a$, que a temperatura inicial seja função do raio, e a superfície do alumínio encontrase em isolamento térmico. O objetivo é avaliar o perfil de temperaturas no cilindro oco em pequenos tempos. 


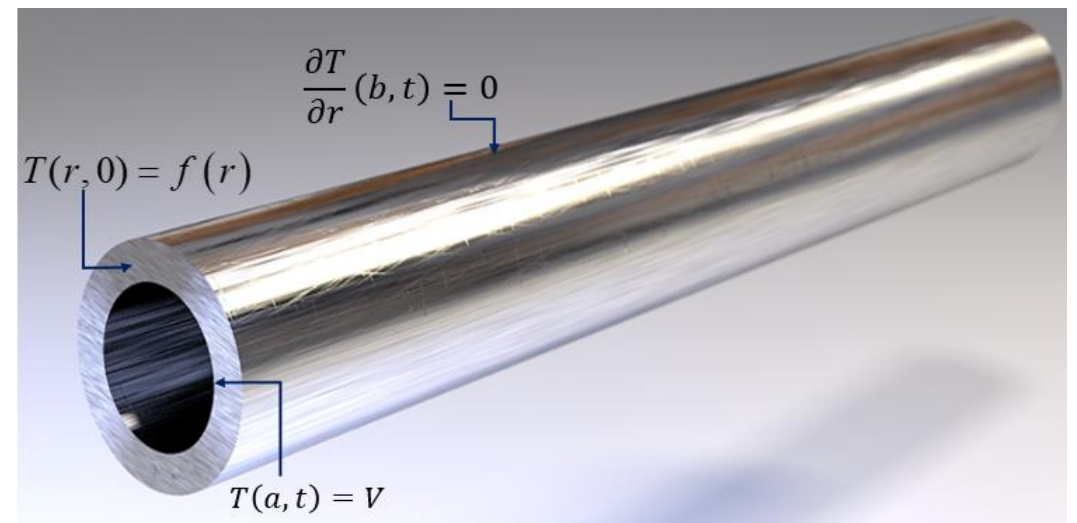

Figura 5. Cilindro oco de alumínio com isolamento térmico na superfície externa.

A formulação matemática para a temperatura no cilindro oco de alumínio é dada por

$$
\begin{aligned}
& \frac{\partial^{2} T}{\partial r^{2}}+\frac{1}{r} \frac{\partial T}{\partial r}=\frac{1}{\kappa} \frac{\partial T}{\partial t}, \quad a \leq r<b \quad t>0 . \\
& C F 1: T(a, t)=V, \quad t>0 \\
& C F 2: \frac{\partial T}{\partial r}(b, t)=0, \quad t>0 \\
& \text { C.I: } T(r, 0)=f(r), \quad a \leq r \leq b .
\end{aligned}
$$

Fazendo a translação, $\varphi=T-V$ tem-se das eqs (60) e (61)

$$
\begin{aligned}
& \frac{\partial^{2} \varphi}{\partial r^{2}}+\frac{1}{r} \frac{\partial \varphi}{\partial r}=\frac{1}{\kappa} \frac{\partial \varphi}{\partial t}, \quad a \leq r<b \quad t>0 . \\
& C F 1: \varphi(a, t)=0, \quad t>0 \\
& C F 2: \frac{\partial \varphi}{\partial r}(b, t)=0, \quad t>0 \\
& \text { C.I }: \varphi(r, 0)=f(r)-V=g(r), \quad a \leq r \leq b .
\end{aligned}
$$

Da eq (12) tem-se $\varphi(r, t)=G(r) H(t)$. Substituindo $\varphi(r, t)$ na eq (62) obtém-se uma equação diferencial na variável $t$ por meio da eq (14), cuja solução é dada pela eq (16), e a equação de Bessel dada pela eq (15), cuja solução pode ser obtida da eq (17) na forma $G(r)=c_{2} J_{0}(\lambda r)+c_{3} Y_{0}(\lambda r)$

Desde que $\varphi(r, t)=G(r) H(t)$ e $G(r)=c_{2} J_{0}(\lambda r)+c_{3} Y_{0}(\lambda r)$ usando a condição CF1 dada pela eq (63) resulta $c_{3}=-c_{2} \frac{J_{0}\left(\lambda_{n} a\right)}{Y_{0}\left(\lambda_{n} a\right)}$, onde $Y_{0}\left(\lambda_{n} a\right) \neq 0$ e com isso, zero não é um autovalor de $Y_{0}$, pois as funções de Bessel de segunda espécie são indefinidas para um argumento nulo. Portanto, resulta

$$
G(r)=C_{4}\left[J_{0}(\lambda r) Y_{0}(\lambda a)-J_{0}(\lambda a) Y_{0}(\lambda r)\right]=C_{4} U_{0}(\lambda r) .
$$

Da condição CF2 dada pela eq (63) e da eq (64) tem-se a equação transcendental dada por 


$$
\left.\frac{d U_{0}(\lambda r)}{d \lambda}\right|_{r=b}=0 \Rightarrow J_{1}(\lambda b) Y_{0}(\lambda a)-J_{0}(\lambda a) Y_{1}(\lambda b)=0 \text {. }
$$

Assim, para cada autovalor não nulo da eq (65) tem-se uma autofunção $U_{0}(\lambda r)$. A solução $\varphi$ é obtida como na eq (20) trocando $G_{0}\left(r, \lambda_{n}\right)$ por $U_{0}\left(\lambda_{n} r\right)$, isto é,

$$
\varphi(r, t)=\sum_{n=1}^{\infty} c_{n} e^{-\kappa \lambda^{2} t} U_{0}\left(r \lambda_{n}\right)
$$

onde os coeficientes $C_{n}$ podem ser obtidos da C.I dada pela eq (63) na forma

$$
\varphi(r, 0)=g(r)=\sum_{n=1}^{\infty} C_{n} U_{0}\left(\lambda_{n} r\right)
$$

sendo $C_{n}=\frac{1}{N\left(\lambda_{n}\right)}=\int_{a}^{b} r g(r) U_{0}\left(\lambda_{n} r\right) d r$. Das eqs (66) e (67) e da translação $\varphi=T-V$, tem-se

$$
T(r, t)=V+\sum_{n=1}^{\infty}\left\{\frac{\int_{a}^{b} r(f(r)-V) U_{0}\left(\lambda_{n} r\right) d r}{\int_{a}^{b} r U_{0}^{2}\left(\lambda_{n} r\right) d r}\right\} U_{0}\left(\lambda_{n} r\right) e^{-\kappa_{2} \lambda_{n}^{2} t},
$$

onde $\lambda_{n}$ são as raízes positivas da equação $\left.\frac{d U_{0}(\lambda r)}{d \lambda}\right|_{r=b}=0$.

A Fig.6 mostra o perfil transiente de temperatura no cilindro oco de alumínio sujeito as condições: $T(a, t)=V=400^{\circ} C, T(r, 0)=V_{0}=30^{\circ} C$. e $\kappa_{2}=80 \times 10^{-6} \mathrm{~m}^{2} / \mathrm{s}$. A condutividade térmica do alumínio $K_{2}=190 \frac{W}{m \cdot{ }^{\circ} C}$ não foi utilizada devido à presença da condição CF3 dada pela eq (61).

Os parâmetros dependentes do tempo foram multiplicados por 60, para obter a resposta em minutos. Os perfis de temperaturas da Figura 6(a) mostram uma leve perturbação da condição inicial. Segundo Carslaw e Jaeger (2011, p. 198, 330) essa anomalia é devida aos pequenos valores de $\frac{\kappa t}{a^{2}} \leq 0.02$ que podem tornar lenta a convergência da série exponencial envolvida na solução analítica quando são utilizados pequenos valores de tempo, em particular em geometrias cilíndricas. 

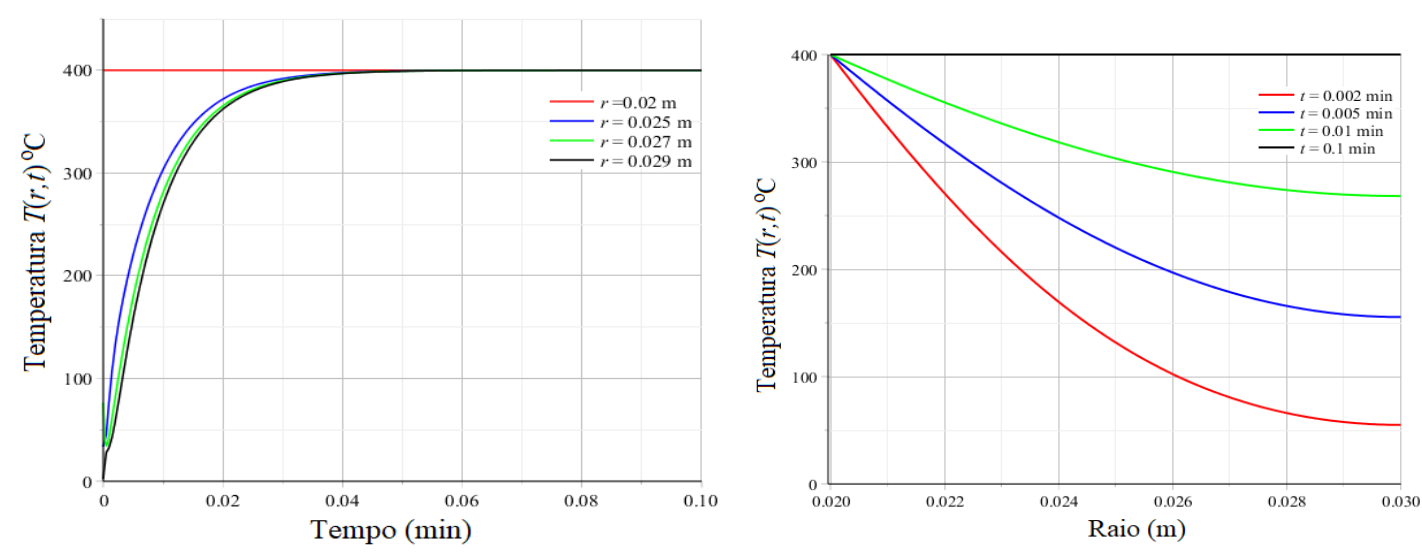

Figura 6. Perfil de temperaturas no cilindro: (a) raios fixados e (b) raio variando.

Nesse problema foram utilizadas as seguintes raízes obtidas da eq (65) dadas por: $\lambda_{1}=144.494, \lambda_{2}=467.239$ e $\lambda_{3}=783.006$.

Nos três casos aqui analisados nesse artigo, procuramos enfatizar detalhadamente as técnicas usadas para as soluções analíticas encontradas e que não foram objetos de estudo de autores como Carslaw e Jaeger (2011) e Hahn e Özisik (2012). Tais soluções podem ser usadas para a validação de soluções aproximadas resultante de uso de métodos numéricos.

\section{Conclusão}

Três casos de distribuição de temperaturas em cilindros circulares infinitos de importância prática foram estudados com a transformada de Laplace, e o método de separação de variáveis. Por se tratar de cilindros finos e longos, perturbações nas soluções acontecem notadamente para pequenos valores de tempo. Em particular, o caso III por envolver uma região de fina camada de material, pode ser notado um pequeno distúrbio em algumas posições do raio com relação à condição inicial. As soluções analíticas incorporam séries exponenciais negativas no tempo, e apesar dos pequenos valores utilizados no tempo, poucos termos da série foram necessários para a obtenção de perfis de temperaturas nos cilindros analisados. As aproximações para esses perfis não foram confrontadas com resultados experimentais em bancadas, laboratórios ou mesmo soluções numéricas, o que seria interessante, já que os mesmos poderiam ser adotados em aplicações em engenharia que exigissem um controle de temperatura do material submetido a um processo de calor, com condições iniciais e de contorno mais complexas preestabelecidas nessa geometria.

\section{Referências}

ARAÚJO, J. C.; MÁRQUEZ, R. G. Problema de Buckley e Leverett: aproximação de funções para a estimativa da saturação de choque. C.Q.D.- Revista Eletrônica Paulista de Matemática, Bauru, v. 16, p. 163-180, dez. 2019. Disponível em: https://www.fc.unesp.br/Home/Departamentos/Matematica/revistacqd2228/v16a10-problema-de-buckley-....pdf. Acesso em: 12 dez. 2019. 
BIEZUNER, R. J. Equações diferenciais parciais I/II. [Belo Horizonte]: UFMG, 2010. Notas de Aula. Disponível em: http://arquivoescolar.org/bitstream/arquivo-e/151/1/edp.pdf. Acesso em: 10 dez. 2018.

CARSLAW, H. S.; JAEGER, J. C. Conduction of heat in solids. 2nd ed. Oxford: Clarendon Press, 2011.

CHURCHILL, R. V. Operational mathematics. 3rd ed. New York: McGraw-Hill, 1972.

CINELLI, G. An extension of the finite hankel transform and applications. International Journal of Engineering Science. v. 3, p. 539-559, out. 1965.

CRANK, J. The mathematics of diffusion. 2nd ed. Oxford: Clarendon Press, 2011.

DAVIS, H. F. Fourier series and orthogonal functions. New York: Dover Publications, 1963.

DEBNATH, L.; BHATTA, D. Integral transforms and their applications. 3rd ed. New York: Chapman \&Hall/CRC, 2015.

FOX, R.W.; PRITCHARD, P. J.; MCDONALD, A. T. Introdução à mecânica dos fluidos. Tradução e revisão técnica de Ricardo Koury e Luiz Machado. 7. ed. Rio de Janeiro: LTC, 2015.

HAHN, D. W.; ÖZISIK, M. N. Heat conduction. Hoboken: John Wiley \& Sons, 2012.

INCROPERA, F. P.; DEWITT, D. P.; BERGMAN, T. L.; LAVINE, A, S. Fundamentos de transferência de calor e de massa. Tradução e revisão técnica Eduardo Mach Queiroz e Fernando Luiz Pellegrini Pessoa. 6. ed. Rio de Janeiro: LTC, 2008.

RIBEIRO, C. da S. A influência térmica na dinâmica das membranas celulares: uma contribuição na conservação de Steindachneridion parahybae (Siluriformes: Pimelodidae), uma espécie de peixe ameaçada de extinção. 2012. 194f. Tese (Doutorado em Ciências) - Universidade de São Paulo, São Paulo, 2012.

SOTOMAYOR, J. Lições de equações diferenciais ordinárias. Rio de Janeiro: IMPA, 1979. (Projeto Euclides).

TRIM, D. W. Applied partial differential equations. Boston: PWS-Kent, 2013.

ZILL, D. G.; WRIGHT, W. S.; CULLEN, M. R. Matemáticas avanzadas para ingeniería. Traducido Erika Jasso Hernan D’Bourneville/Carlos Roberto Cordero Pedraza/Gabriel Nagore Cazares. 4. ed. McGRAW-HILL/Interamericana Editores, 2011. 